\title{
Submillimeter-Wave Oscillations in Recessed InGaAs/InAlAs Heterostructures: Origin and Tunability
}

\author{
S. PÉrez*, J. Mateos And T. GonzÁlez \\ Dpto. Física Aplicada, Universidad de Salamanca, Plaza de la Merced s/n, 37008 Salamanca, Spain
}

\begin{abstract}
By means of an ensemble Monte Carlo simulator, the appeareance of THz oscillations in InAlAs/InGaAs slot diodes is predicted when the applied bias exceeds the threshold for intervalley transfer. Such high frequency is attained by the presence of a Gunn-like effect in the recess-to-drain region of the device channel whose dynamics is controlled by ballistic $\Gamma$ valley electrons. In this work we explain the mechanism at the origin of this effect and also the influence of the bias conditions, $\delta$-doping, recess-to-drain distance and recess length on the frequency of the ultrafast Gunn-like oscillations. The simulations show that a minimum value for the $\delta$-doping is necessary to have enough carrier concentration under the recess and allow the oscillations to emerge. Finally, we show that shortening the devices (small recess and recess-to-drain lengths) increases the oscillation frequency, so provides an interesting frequency tunability of this $\mathrm{THz}$ source.
\end{abstract}

PACS: 85.30.Fg, 07.57.Hm, 73.40.Kp

\section{Introduction}

Submillimeter waves refer to wavelengths ranging between $1 \mathrm{~mm}$ and $100 \mu \mathrm{m}$, corresponding to a frequency range from $300 \mathrm{GHz}$ (higher part of microwave systems) to $3 \mathrm{THz}$ (far-infrared technology). Therefore, the sources of this region of the electromagnetic spectrum are at the limits of electronics on the one hand and optical systems on the other. This fact has made the progress of terahertz technologies depend strongly on the development of compact, powerful and tunable $\mathrm{THz}$ sources working at room temperature, hardly existing nowadays.

In this contribution we propose a source of terahertz radiation obtained from an ultrafast Gunn-like effect happening in recessed InGaAs/InAlAs heterostructures in which high electron mobility transisitors (HEMTs) are based $[1,2]$. From the results of ensemble Monte Carlo simulations of the devices [3] a detailed explanation of the origin and frequency tunability of the oscillations will be presented.

\section{Device topology and Monte Carlo approach}

The layer structure of the simulated slot diodes is similar to that used for the fabrication of InP-based HEMTs $[1,4]$ : a $10 \mathrm{~nm}$ InGaAs cap layer, heavily doped with $N_{\mathrm{D}}=6 \times 10^{18} \mathrm{~cm}^{-3}$, followed by a $10 \mathrm{~nm} \operatorname{In}_{0.52} \mathrm{Al}_{0.48} \mathrm{As}$ barrier layer, a $\delta$-doping layer of $6 \times 10^{12} \mathrm{~cm}^{-2}$ and a $3 \mathrm{~nm}$ undoped $\mathrm{In}_{0.52} \mathrm{Al}_{0.48}$ As spacer, to continue with a $15 \mathrm{~nm} \mathrm{In}_{0.70} \mathrm{Ga}_{0.30}$ As channel placed over a $200 \mathrm{~nm}$ InAlAs buffer. The geometry of the device that we will use as a reference (Fig. 1) has a source-to-recess distance

\footnotetext{
* corresponding author; e-mail: susana@usal.es
}

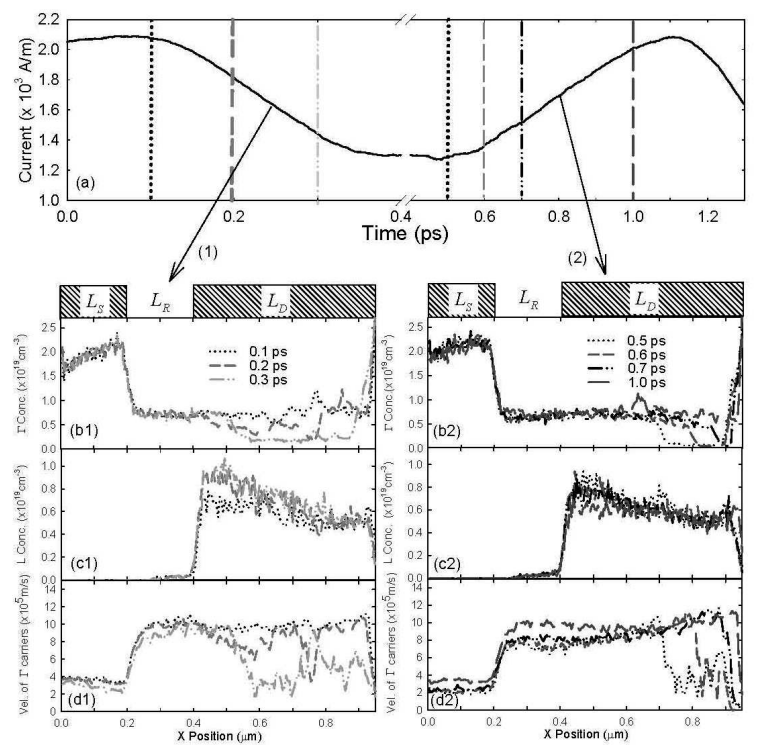

Fig. 1. (a) Time-varying current. (b) Profile of $\Gamma$ -valley (upper part) and (c) $L$-valley (medium part) electron concentrations and (d) $\Gamma$-valley velocity (lower part) in the channel of the heterostructure at different time moments within one period of the oscillation (left side for decreasing current (b1, c1, d1) and right side for increasing current $(\mathrm{b} 2, \mathrm{c} 2, \mathrm{~d} 2))$ for a bias $V_{\mathrm{DS}}=1.0 \mathrm{~V}$ and $L_{\mathrm{S}}=200 \mathrm{~nm}, L_{\mathrm{R}}=200 \mathrm{~nm}$ and $L_{\mathrm{D}}=550 \mathrm{~nm}$.

$\left(L_{\mathrm{S}}\right)$ of $200 \mathrm{~nm}$, a recess length $\left(L_{\mathrm{R}}\right)$ of $200 \mathrm{~nm}$ and a recess-to-drain distance $\left(L_{\mathrm{D}}\right)$ of $550 \mathrm{~nm}$.

Simulations are performed by using an ensemble Monte Carlo model self-consistently coupled to a 2D Poisson solver. In the simulator the contacts are placed vertically adjacent to the different materials. Therefore non-uniform potential and concentration profiles are consid- 
ered along these contacts to correctly simulate real top electrodes. The effect of degeneracy is accounted for by locally using a simple rejection technique, where electron heating and nonequilibrium screening effects are introduced by means of the local electron temperature. No other quantum effects are considered in the simulation in order to have reasonable CPU times. The validity of this approach, especially under high-field conditions, and that of the whole MC model has been confirmed in previous works [4]. In order to detect the presence of ultrafast Gunn oscillations, special attention is devoted to the calculation of the current noise spectra due to their extreme sensitivity to microscopic features of carrier dynamics and the possibility to easily perform a frequency analysis of the electrical fluctuations. Moreover, taking advantage of the time-domain microscopic description given by the MC simulations, a detailed study of the instantaneous profiles of velocity, concentration, valley occupation, potential and electric field within one period of the oscillation is carried out.

\section{Results}

When a dc voltage between the drain and source terminals is applied to this diode, depending on the bias range, two different behaviours can be observed. For lower voltages $\left(V_{\mathrm{DS}}<0.6 \mathrm{~V}\right)$, non-coherent, small amplitude current oscillations appear, caused by plasma effects. However, when high voltages are applied $\left(V_{\mathrm{DS}}>0.6 \mathrm{~V}\right)$, clear coherent oscillations appear in the current, Fig. 1a, with a frequency around $1 \mathrm{THz}$. This paper is focused on the investigation of the origin and tuning of the latter $\mathrm{THz}$ current oscillations. It must be noted that $\Gamma-L$ energy separation in the InGaAs channel is $0.61 \mathrm{eV}$. Therefore, as for classical Gunn oscillations, the oscillatory behavior of the current should be somehow related to intervalley transfer mechanisms that push carriers into the upper satellite valleys. However, the creation and annihilation of space-charge domains in the slot diodes under study exhibit important differences with respect to the behavior of classical Gunn devices. Firstly, the active region for the formation and drift of the field inhomogeneity is not the total cathode-to-anode distance, but only the recess-to-drain region. Indeed, the value of the electric potential at the drain edge of the recess is responsible for the generation and maintenance of the oscillations thanks to the modulation of its value along a period.

To understand the origin of the oscillation it is interesting to observe the profile of the $\Gamma$ and $L$ electron concentrations in the channel at different times within one period, as shown in Fig. 1b and c, respectively, for an applied voltage of $1.0 \mathrm{~V}$.

The maximum value of the current (at the time $0.1 \mathrm{ps}$ ) is reached when the $\Gamma$-concentration along the recess-to-drain region is nearly constant; no depleted zone is observed. At this time the most resistive part of the device is the low concentration region under the recess. In this situation, if $V_{\mathrm{DS}}$ is high enough, a voltage drop larger than the $\Gamma-L$ intervalley energy separation takes place under the recess and, as a consequence, an increasing amount of electrons starts to jump to the $\mathrm{L}$ valley near the drain edge of the recess. As the $L$ electrons are very slow as compared to those in the $\Gamma$ valley, the $\mathrm{L}$ valley electron population at the recess-drain region starts to increase (see Fig. 1c1 for times $0.2 \mathrm{ps}$ and $0.3 \mathrm{ps}$ ). Simultaneously, at the right side of the region with enhanced $L$-valley concentration a depleted zone of $\Gamma$ carriers appears (see Fig. 1c1 for times $0.2 \mathrm{ps}$ and $0.3 \mathrm{ps}$ ) due to the fast drift of $\Gamma$ electrons that are not replaced at this position. The depleted $\Gamma$ electron region is widened as the time goes by, producing a decrease of the current, as observed for the time interval between 0.1 ps to around $0.3 \mathrm{ps}$. This depleted region is more and more resistive and absorbs an increasing fraction of the applied potential, thus creating a high electric field domain. Indeed, for 0.1 ps a very small fraction of the applied potential drops along the recess-drain region, but as the depleted region increases it absorbs a higher potential drop, reaching a situation in which the value of the electric potential at the drain edge of the recess is lower than the $\Gamma-L$ intervalley energy separation. Under these conditions the carriers injected into the drain region will be mostly very fast electrons that stay at the $\Gamma$ valley, which progressively fill the depleted region previously described (Fig. 1c2, times between $0.3 \mathrm{ps}$ and $1.0 \mathrm{ps}$ ) and produce an increase of the current (Fig. 1a) until the uniform carrier concentration of the starting conditions is achieved.

In Fig. $1 \mathrm{~d} 1$ and $\mathrm{d} 2$ it can be observed that these $\Gamma$ valley electrons injected into the drain region exhibit a very high velocity (as high as $10 \times 10^{7} \mathrm{~cm} / \mathrm{s}$ ) due to the low effective mass of InGaAs and the high electric field in the recess region, so that they move quasiballistically along the recess-to-drain region. Since the period of the oscillations comes mainly from the time of drift of the high field domain in the recess-drain region and its velocity is given by that of $\Gamma$ electrons, such high velocity is the main responsible for attaining oscillations at $\mathrm{THz}$ frequencies. It is important to remark that this ultrahigh-frequency nonstationary phenomenon is rather different from the classical Gunn effect, which is based on the propagation of a dipolar domain that always travels at the electron saturation velocity, slightly higher than $1 \times 10^{7} \mathrm{~cm} / \mathrm{s}$ in InGaAs; therefore, a much slower process.

\subsection{Dependence on the applied voltage}

Figure 2 (solid line) shows how the oscillation frequency decreases with $V_{\mathrm{DS}}$. Although the velocity of $\Gamma$ electrons is nearly constant with the bias, for voltages just above the threshold for the onset of the oscillations $L$ valley electrons are not injected just at the drain side of the recess, but slightly inside the drain region, thus decreasing the distance the depletion/filling region has to travel. Consequently, higher oscillation frequencies are obtained. 


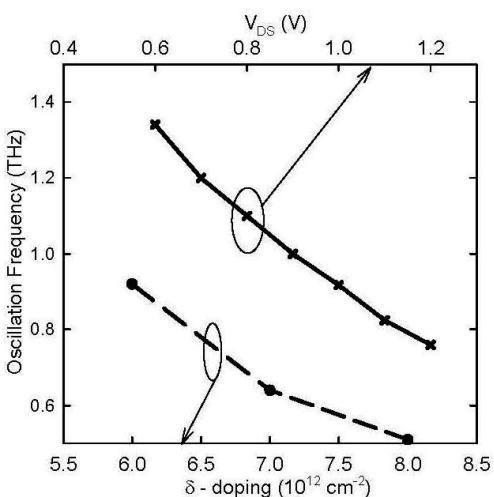

Fig. 2. Oscillation frequency as a function of $V_{\mathrm{DS}}$ (solid line) for a $\delta$-doping $=6 \times 10^{12} \mathrm{~cm}^{-2}$, and as a function of the $\delta$-doping (dashed line) for $V_{\mathrm{DS}}=1.0 \mathrm{~V} . L_{\mathrm{S}}=$ $200 \mathrm{~nm}, L_{\mathrm{R}}=200 \mathrm{~nm}, L_{\mathrm{D}}=550 \mathrm{~nm}$.

\subsection{Dependence on the $\delta$-doping}

The presence of a minimum amount of carriers in the channel under the recess is required for the onset of the current oscillations. In fact, oscillations do not take place in our reference device for $\delta$-dopings lower than $6 \times 10^{12} \mathrm{~cm}^{-2}$. However, if the $\delta$ doping is further increased, the oscillation frequency is degraded (Fig. 2, dashed line) because of the less efficient focalisation of the potential drop by the recess and, as a consequence, the velocity of electrons injected into the drain region (and also that of the domain drift) decreases.

\subsection{Dependence on the recess-to-drain $\left(L_{\mathrm{D}}\right)$ and recess $\left(L_{\mathrm{R}}\right)$ lengths}

In Fig. 3 the value of the oscillation frequency $f_{0}$ is plotted as a function of $L_{\mathrm{D}}$ when the bias is fixed to $1.0 \mathrm{~V}$ (solid line) and $L_{\mathrm{S}}=L_{\mathrm{R}}=200 \mathrm{~nm}$ are kept constant. As expected, by reducing the length of the recess-drain region the oscillation frequency increases because the transit time of the high electric field domain is shortened. However, for the shorter devices the increase of $f_{0}$ does not follow the expected $1 / \mathrm{L}_{\mathrm{D}}$ dependence. This deviation from the simple transit time criterion for $L_{\mathrm{D}}<550 \mathrm{~nm}$ is mainly a consequence of the increase of $L$-valley population at the drain side of the recess (due to a sharper voltage drop at that point), which affects the dynamics of the depletion/filling mechanism.

When the recess length increases (Fig. 3, dashed line), while $L_{\mathrm{S}}=200 \mathrm{~nm}$ and $L_{\mathrm{D}}=550 \mathrm{~nm}$ are kept constant, the oscillation frequency decreases. A longer recess has a smaller capability to concentrate the potential drop necessary to sharply start the intervalley transfer. As a consequence, an important amount of electrons jump to the $\mathrm{L}$ valley before reaching the drain side of the recess, degrading the modulation of the current and absorbing a large part of the applied voltage. The velocity overshot associated with ballistic $\Gamma$ electrons is quite reduced, and the electrons injected into the recess-drain region exhibit lower velocities.

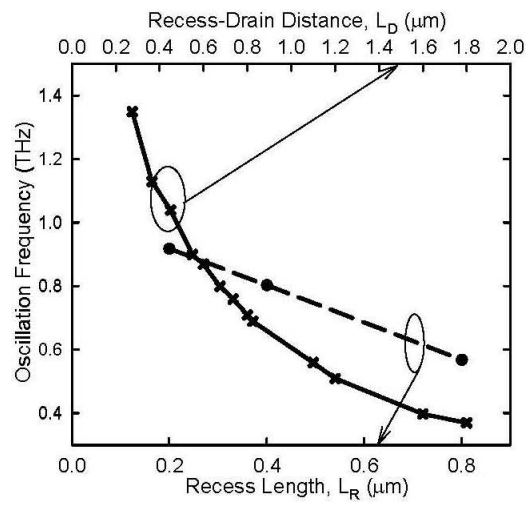

Fig. 3. Oscillation frequency as a function of the recess-drain distance (solid line) with $L_{\mathrm{R}}=200 \mathrm{~nm}$, and as a function of the recess length (dashed line) with $L_{\mathrm{D}}=550 \mathrm{~nm}$, for $V_{\mathrm{DS}}=1.0 \mathrm{~V} . L_{\mathrm{S}}=200 \mathrm{~nm}$.

\section{Acknowledgments}

This work was supported by the Junta de Castilla y Leon, under projects GR270 and SA019A08, by the Dirección General de Investigación (MEC) and FEDER through project TEC2010-15413, and by the European Commission, ROOTHz project ICT-2009-243845.

\section{References}

[1] J. Mateos, T. González, D. Pardo, V. Hoël, A. Cappy, IEEE Trans. Electron Dev. 47, 1950 (2000).

[2] N. Dyakonova, A. El Fatimy, J. Łusakowski, W. Knap, M.I. Dyakonov, M.A. Poisson, E. Morvan, S. Bollaert, A. Shchepetov, Y. Roelens, Ch. Gaquiere, D. Theron, A. Cappy, Appl. Phys. Lett. 88, 141906 (2006).

[3] S. Pérez, T. González, D. Pardo, J. Mateos, J. Appl. Phys. 103, 094516 (2008).

[4] J. Mateos, T. González, D. Pardo, V. Hoel, A. Cappy, IEEE Trans. Electron. Dev. 47, 1950 (2000). 